\title{
Multiple Source Localization for Partial Discharge Monitoring in Electrical Substation
}

\author{
Y. Zhang ${ }^{1}$, D. Upton ${ }^{1}$, A. Jaber ${ }^{1}$, H. Ahmed ${ }^{1}$, U. Khan $^{1}$, B. \\ Saeed $^{1}$, P. Mather ${ }^{1}$, \\ ${ }^{1}$ Department of Engineering \& Technology, \\ University of Huddersfield, Huddersfield HD1 3DH, UK \\ y.zhang@hud.ac.uk
}

\begin{abstract}
This paper studies novel localization methods of multiple partial discharge sources in electrical substations. The three compressive sensing algorithms of Orthogonal Matching Pursuit (OMP), Homotopy technique, and Dichotomous coordinate descent (DCD) are presented. The simulation results demonstrate excellent performance with the compressive sensing methods.
\end{abstract}

Keywords-partial discharge; localization; multiple sources; comprssive sensing

\section{INTRODUCTION}

Radio frequency (RF) measurements have been extensively applied in substations for in-service condition monitoring of partial discharge (PD) activity. A radiometric wireless sensor monitoring network has been presented to make the whole substation coverage for PD online measurement and control [1]. One of the main advantages of radiometric wireless sensor network (WSN) measurement is to implement PD localization at low cost in substation. The three methods of time-of-arrival (TOA), time-difference-of arrival (TDOA) and angle-of-arrival (AOA) have drawn considerable research attentions in PD location areas [2-4]. Unlike TOA, TDOA and AOA, the RSSI (received signal strength indicator) based source localization does not require time synchronization or use of an antenna array, and make it cost effective [5]. Antilog function is used to convert RSSI values to distance values. However, small RSSI variation in decibel form leads to large variation in estimated distance, and passloss index may vary with electromagnetic environment in electrical substation. These localization methods are typically used to estimate a single PD location.

In the paper, novel RSSI-based localization methods are presented for multiple PD sources using compressive sensing. The measured data of PD strength at radiometric wireless nodes (RNs) are collected to and to perform localization at the central controller. The localization estimates can be obtained by searching for the sparsest solution of an under-determined linear system of equations.

\section{LocAtion Method UsING COMPRESSIVE SENSING}

The proposed radiometric WSN integrates RF monitoring technology into wireless sensor network to form a continuous

\author{
P. Lazaridis ${ }^{1}$, R. Atkinson ${ }^{2}$, M. F. Q Vieira ${ }^{3}$ and I.A. Glover ${ }^{1}$ \\ ${ }^{2}$ Deptartment of Electronic and Electrical Engineering, \\ University of Strathclyde, Glasgow G1 1XW, UK \\ ${ }^{3}$ Department of Electrical Engineering, University Federal \\ de Campina Grande, Campina Grande, Brazil
}

real time condition monitoring system over coverage of the whole substation.

The key idea of the proposed localization is to reconstruct a sparse signal from a set of appropriate RSSI measurements by applying compressive sensing. A grid based representation of the substation space is used to create a finite set of possible positions. The localization algorithms consist of two steps of calibration and runtime phase. During calibration phase, each RN acquires signal strength measurements from a RN for each cell of the grid in order to construct a map of signatures of the physical space in substation. In the runtime phase, each RN receives RSSI measurements in order to estimate the average received PD signal and sends it to the central unit. The central controller collects the RSSI fingerprints from the RNs and applies the Compressive sensing localization algorithms in order to estimate the position of PD sources.

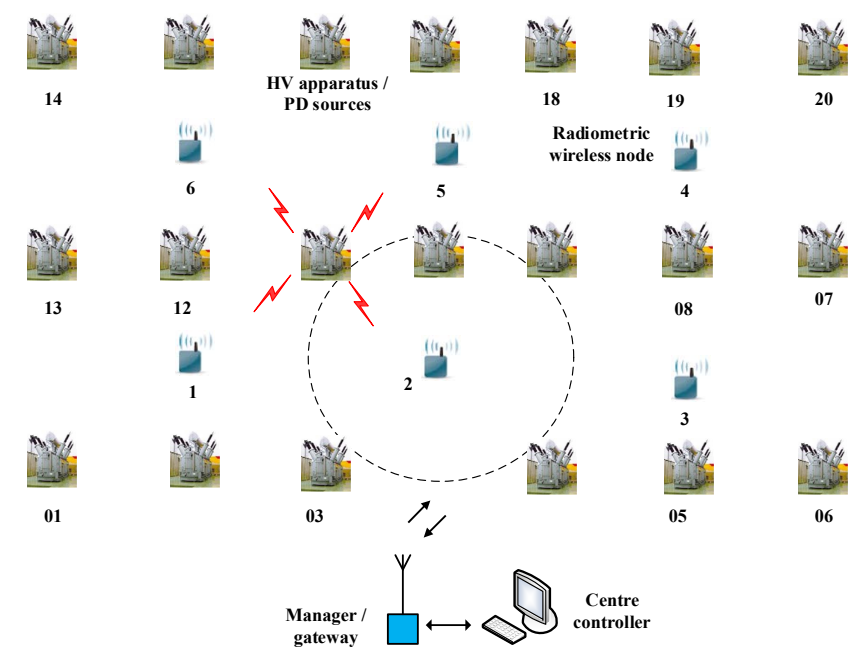

Fig. 1. The layout of PD WSN at a substation: 1 denotes PD electromagnetic signals, - HV apparatus, -radiometric node

We assume that there is $M$ radiometric wireless nodes (RNs) used to monitoring the PD activities, and the whole two dimension substation space is discretized into $N$ grid cells, which are potentially PD sources, shown in Fig. 1. By calibration phase, an observation matrix is formed as follows, 


$$
A=\left[\begin{array}{cccc}
P_{1,1} & P_{1,2} & \cdots & P_{1, N} \\
P_{2,1} & P_{2,2} & \cdots & P_{2, N} \\
\cdots & \cdots & \cdots & \cdots \\
P_{M, 1} & P_{M, 2} & \cdots & P_{M, N}
\end{array}\right]
$$

Where, $P_{i, j}$ denotes the measured power at $i$ th RN coming from the $j$ th cell during calibration phase. $x \in R_{N \times 1}$ an unknown vector with $k$ non-zero number (PD sources) that we would like to recover. $y \in R_{M \times 1}$ denotes the received power vector at RNs during runtime operation. We have to solve the underdetermined linear equation below,

$$
y=A x
$$

There are several approaches to solve the underdetermined system in (1): 1. Greedy selection algorithms such as Orthogonal Matching Pursuit (OMP) [6], 2. Homotopy technique [7], 3. Dichotomous coordinate descent (DCD) [8].

\section{A. Greedy Algorithms / Orthogonal Matching pursuit}

Greedy algorithms are sub-optimal iterative algorithms that attempt to solve a under-determined matrix problem by successively adding new atoms into a sparse approximation with the objective of minimizing the new residual. Orthogonal Matching pursuit (OMP) is an iterative greedy algorithm that selects at each step the dictionary element best correlated with the residual part of the signal. Then it produces a new approximant by projecting the signal onto those elements which have already been selected [6]. Procedure of OMP is as follows:

1. Initialize the residual $r 0=y$, the index set $\Lambda 0=[]$, and the iteration counter $t=1, \Lambda m$ contains $m$ elements from $\{1$, ..., $d\}$.

2. Find the index $\lambda t$ that solves the easy optimization problem

$$
\lambda_{t}=\arg \max _{j=1, \cdots, N}\left|\left\langle r_{t-1}, \varphi_{j}\right\rangle\right|
$$

If the maximum occurs for multiple indices, break the tie deterministically.

3. Augment the index set and the matrix of chosen atoms: $\Lambda t=\Lambda t-1 \cup \lambda t$ and $A t=\left[\begin{array}{ll}A t-1 & \varphi_{\lambda_{t}}\end{array}\right]$. We use the convention that is $A 0$ is an empty matrix.

4. Solve a least squares problem to obtain a new signal estimate:

$$
x_{t}=\arg \min _{x}\left\|y-A_{t} x\right\|_{2}
$$

5. Calculate the new approximation of the data and the new residual

$$
r_{t}=y-A_{t} x_{t}
$$

6. Increment $t$, and return to Step 2 if $t<m$.

\section{B. Homotopy Based Location Technique}

By classical ideas in convex analysis, a necessary condition for $x$ to be a minimizer of $J \tau(x)$ is that [7]

$0 \in \partial x J \tau(x)$,i.e. the zero vector is an element of the subdifferential of $J \tau$ at $x$.

$$
\partial x J \tau(x)=-A T(y-A x)+\tau \partial\|x\|_{1}
$$

A necessary condition for $x$ to be a minimizer of $\partial x J \tau(x)$ is that the subdifferential of $J \tau(x)$ is zero.

$$
\partial\|x\|_{1}= \begin{cases}\operatorname{sign}(x), & x \neq 0 \\ \in[1,-1], & x=0\end{cases}
$$

Let $I=\{i: x(i), 0\}$ denote the support of $x$, and $c=A^{H}(y-A x)$ denote the correlations between the dictionary matrix and the residue. Then equation (2.4) can be written equivalently as

$$
c(I)=\tau \operatorname{sign}(x(I))
$$

and

$$
|c(I c)| \leq \tau
$$

In other words, the residual correlations on the support $I$ must all have magnitude equal to $\tau$, while

residual correlations off the support must have magnitude less than or equal to $\tau$. Hence, the active set $I$ satisfies

$$
I=\{j:|c(j)|=\|c\| \infty=\tau\}
$$

The Homotopy algorithm starts with an initial solution $x 0$ $=0$, and computes iterative solution estimate $x l, l=1,2, \ldots$

$$
x l=x l-1+\gamma l d l
$$

This magnitudes of residual correlations decline equally on the active set. We can find $\gamma l$ by solving

$$
\begin{gathered}
\left|A^{T}{ }_{l}(y-A I(x I+\gamma l d l))\right|=\tau-\gamma l \\
\gamma_{l}=\frac{\tau \pm c_{l}(i)}{1 \pm a_{i}^{T} A_{I} d_{l}}
\end{gathered}
$$

thus, smallest positive step is

$$
\gamma_{l}=\min \left\{\frac{\tau \pm c_{l}(i)}{1 \pm a_{i}^{T} A_{l} d_{l}}\right\}
$$

a direction $d l$ may be updated by solving

$$
A_{I}^{T}(y-A I(x I+\gamma l d l))=(\tau-\gamma l) \operatorname{sign}(x I+\gamma l d l)
$$

thus

$$
\begin{array}{r}
A_{I}^{T} A I d l=\operatorname{sign}(x I+\gamma l d l) \\
\operatorname{sign}(x l(i))=\operatorname{sign}(c l(i))[6], \text { so } \\
A_{I}^{T} A I d l=\operatorname{sign}(\operatorname{cl}(I))
\end{array}
$$

\section{DCD Algorithm}

When the sparse level is sufficient, Spars approximate problem can be expressed as,

$$
\min \|x\|_{1} \text {, subject to } A x=y
$$

The above $l 1$ problem leads to Basis Pursuit Denoising (BPDN) optimization problem, ie. the following Lagrangian formulation, also called LASSO problem,

$$
\min \|y-A x\|_{2}^{2} / 2+\tau\|x\|_{1}
$$

where $\tau \geq 0$ is a scalar regularization parameter, balancing the tradeoff between the two terms, namely, sparsity and residual error. The $l 2$-term forces the residual $y-A x$ to be small, whereas the $l 1$-term enforces sparsity of representation. To find $x$ in (18), the following optimization problem is solved,

$$
x_{\tau}=\underset{x}{\arg \min } J_{\tau}(x)
$$


Where, for a positive scalar $\tau$, the cost function, $J_{\tau}(x)$ is given by

$$
\begin{aligned}
J_{\tau}(x) & =\frac{1}{2}(y-A x)^{H}(y-A x)+\tau \sum_{n=1}^{N}\left|x_{n}\right| \\
& =\frac{1}{2} y^{H} y+\frac{1}{2} x^{H} R x-\Re\left\{b^{H} x\right\}+\tau \sum_{n=1}^{N}\left|x_{n}\right|
\end{aligned}
$$

Where $\quad R=A^{H} A, \quad b=A^{H} y, \quad(\cdot)^{H}$ denotes Hermitian transpose and $\Re(\cdot)$ real part of a complex number. The problem (20) is convex. DCD location algorithm [8] is summarized in TABLE 1.

TABLE 1. DCD ALGORITHM

\begin{tabular}{|r|c|}
\hline Step & Equation \\
\hline & Initialization: $\mathrm{x}=0, \mathrm{r}=\mathrm{b}, \tau=\max _{q}\left|b_{q}\right|, c_{u}=0$, \\
& $L_{\gamma}=1, \delta=\delta_{\max }, \gamma$ is empty \\
\hline 1 & Repeat until $\tau<\tau_{\min }$ or $c_{u}=N_{u}$ or $L_{\gamma}=L_{\max }$ \\
\hline 2 & $\tau \leftarrow \beta \tau, m=1, \alpha=[\delta,-\delta, j \delta,-j \delta]$ \\
\hline 3 & $p=\arg \max _{q}\left|b_{\gamma, q}\right|$ \\
\hline 4 & Repeat until $m=M_{\mathrm{b}}$ \\
\hline 5 & Flag $=0$ \\
\hline 6 & Repeat for $\mathrm{k}=1,2,3,4$ \\
\hline 7 & $h_{p} \leftarrow h_{p}+\alpha_{k}, d \leftarrow d+\alpha_{k} A^{(p)}, b \leftarrow b-\alpha_{k} A^{H} A^{(p)}$ \\
\hline 8 & $p=$ arg max ${ }_{q}\left|b_{\gamma, q}\right|$, Flag $=1, c_{u} \leftarrow c_{u}+1$, break \\
\hline 10 & If Flag $=0$, do \\
\hline 11 & $m=m+1, \delta \leftarrow \lambda \delta, \alpha=[\delta,-\delta, j \delta,-j \delta]$ \\
\hline 12 & test on removing an element in $\gamma$ and \\
\hline 13 & test on adding an element in $\gamma$ and update $\gamma$ \\
\hline & and $L_{\gamma}$ if required \\
\hline
\end{tabular}

\section{Simulation Results}

In this section, we present experimental results that show the compressive sensing algorithms are powerful methods for multiple PD location in substation. Gaussian or sign sparse signals are generated as original sparse signals in dimension $N$ $=256$, namely grid cell number over substation space. Sign signals are generated by taking sign function of Gaussian distribution. We also used Gaussian measurement matrices with measurement length $M=128$, namely RN number. Sparsity levels (nonzero number) are chosen from $k=1$ to $K=$ 80, namely PD source number. We compare the supports between original signal and recovered signal by probability of recovery while the mean squared error (MSE) is used to evaluate the performance of the the proposed algorithm as

$$
\xi=\frac{\left[x-x_{0}\right]^{T}\left[x-x_{0}\right]}{x_{0}^{T} x_{0}}
$$

where $x$ is recovered signal, $x 0$ is ideal sparse signal. All results are obtained on average of 200 independent trials and plotted against sparse level.

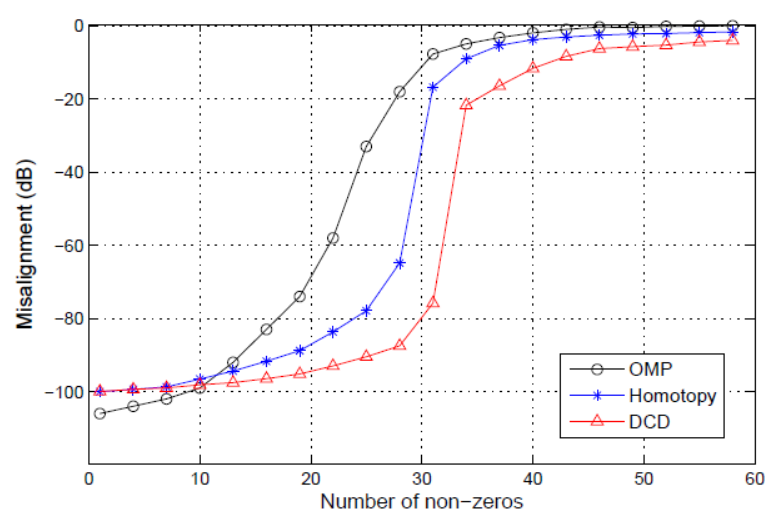

Fig. 2. Mean squared error for sign sparse signal

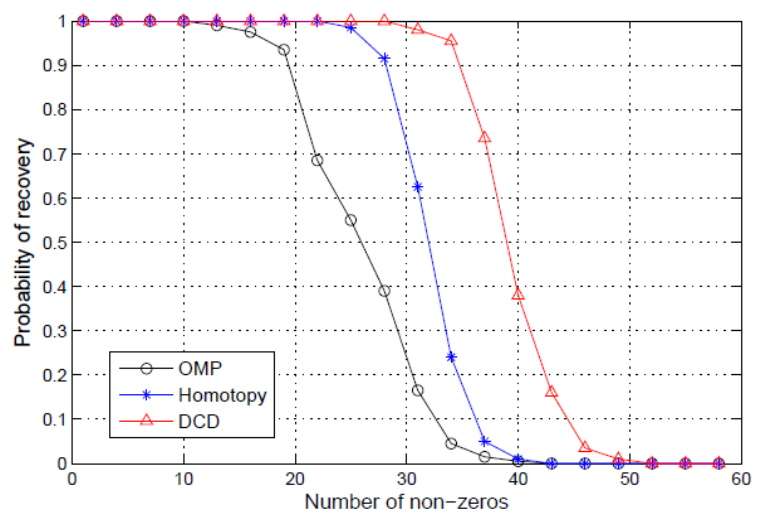

Fig. 3. Probability of recovery for sign sparse signal

Fig. 2 shows the misalignment performance of OMP, Homotopy method and DCD algorithm. Fig. 3 compares recovering probability of the three algorithms. We think that the recovery is successful if the maximum absolute value of difference between elements of $x 0$ and $x$ is less than $10^{-3}$. All three algorithms demonstrate perfect performance for sparsest level. Along with the number of non-zeros increasing, OMP is getting worst and the DCD algorithm keeps best on both misalignment and recovering probability performance. When PD source number $K$ is less than 10, all three compressive sensing algorithms have perfect performance. There is an acceptable performance for $10<K<20$.

\section{CONCLUSION}

The proposed multiple localization method make good performance for PD monitoring of radiometric WSN over coverage of the whole substation by using compressive sensing technique. Due to complex electromagnetic environment in substation, interference noise should be included into the system evaluation for future further research.

\section{ACKNOWLEDGEMENT}

This work was supported by the U.K. Engineering \& Physical Sciences Research Council (EPSRC) [grant number $\mathrm{EP} / \mathrm{J} 015873]$. 


\section{REFERENCES}

[1] Y. Zhang, J. M. Neto, D. Upton, etal, "Radiometer Monitoring System for Partial Discharge Detection in Substation," 2015 URSI Atlantic Radio Science Conference, Gran Canaria, Spain, May 2015.

[2] P. J. Moore, I. Portugues and I. A. Glover, "Radiometric location of partial discharge sources on energized high-voltage plant," IEEE Trans. in Power Delivery, vol. 20, no. 3, pp.2264-2272, July 2005.

[3] I. E. Portugués, P. J. Moore and I. A. Glover, "RF-based partial discharge early warning system for air-insulated substations," IEEE Transactions on Power Delivery, Vov.24, No.1, pp.20-29, January 2009.

[4] H. Hou, G. Sheng and X. Jiang, "Localization algorithm for the PD source in substation based on L-shaped antenna array signal processing,"
IEEE Transactions on Power Delivery, Vol. 30, no. 1, pp.427-479, February 2015.

[5] Y. Xu, J. Zhou and P. Zhang, "RSS-based source localization when path-loss model parameters are unknown," IEEE Communications Letters, Vol. 18, No. 6, pp. 1055-1058, June 2014.

[6] J. Tropp and A. Gilbert, "Signal recovery from random measurements via orthogonal matching pursuit,", IEEE Transactions on Information Theory, vol.12, pp. 4655-4666, 2007.

[7] D. Donoho, and Y. Tsaig, "Fast solution of 11 minimization problems when the solution may be sparse," Research Report, Department of Statistics, Stanford University, 2006.

[8] Y. Zakharov and V. Nascimento, "Homotopy algorithm using dichotomous coordinate descent iterations for sparse recovery," $46^{\text {th }}$ Asilomar Conference on Signal, Systems and Computers, Pacific Grove, USA, Nov. 2012. 